\title{
Frequência dos tipos sanguíneos de gatos domésticos mestiços no município de Campo Grande, Mato Grosso do Sul, Brasil
}

\author{
[Frequency of blood types of crossbred domestic cats in the municipality of Campo Grande, Mato \\ Grosso do Sul, Brazil]
}

\section{"Artigo Científico/Scientific Article"}

\author{
Simone Sorgatto ${ }^{1}$, Bruna Brito de Oliveira ${ }^{1}$, Kelly Cristina da Silva Godoy ${ }^{1 *}$, \\ Tamires Ramborguer Antunes ${ }^{1}$, Luciana Almeida Lacerda ${ }^{2}$, Alda Izabel de Souza ${ }^{1}$
}

\author{
${ }^{1}$ Faculdade de Medicina Veterinária e Zootecnia, Universidade Federal de Mato Grosso do Sul (FAMEZ/UFMS), \\ Campo Grande-MS, Brasil. \\ ${ }^{2}$ Blut's Centro de Diagnósticos Veterinários, Porto Alegre-RS, Brasil. \\ *Autor para correspondência/Corresponding author: E-mail: k.c.s.godoy@gmail.com
}

\begin{abstract}
Resumo
O objetivo deste estudo foi determinar as frequências dos tipos sanguíneos, do sistema $\mathrm{AB}$, de gatos domésticos sem raça definida na cidade de Campo Grande, Mato Grosso do Sul, Brasil. Foram selecionados aleatoriamente 202 animais clinicamente saudáveis e a tipagem sanguínea foi realizada por meio do teste de hemaglutinação em tubo de ensaio. A distribuição da frequência foi de $98,5 \%$ e $1,5 \%$ para gatos dos tipos $\mathrm{A}$ e $\mathrm{AB}$, respectivamente. Gatos do tipo B não foram identificados. Os resultados demostraram que no município de Campo Grande há predomínio de gatos domésticos mestiços do tipo sanguíneo A, contudo a presença de gatos com tipo $\mathrm{AB}$ e a ausência do tipo $\mathrm{B}$, indicam peculiaridade da população escolhida. Estes dados alertam sobre a possibilidade de reações transfusionais adversas e a ocorrência de isoeritrólise neonatal, e contribui para a seleção adequada de doadores em banco de sangue animal.
\end{abstract}

Palavras-Chave: antígenos eritrocitários; imunohematologia sistema AB.

\begin{abstract}
The aim of this study was to determine the AB blood types of mixed breed domestic cats in the city of Campo Grande, Mato Grosso do Sul, Brazil. Twenty-two clinically healthy animals were randomly selected and blood typing was performed using a test tube haemagglutination test. The frequency distribution was $98.5 \%$ and $1.5 \%$ for types $\mathrm{A}$ and $\mathrm{AB}$ cats, respectively. Type $\mathrm{B}$ cats were not identified. The results showed that in the municipality of Campo Grande there is a predominance of crossbred domestic cats of type A, but the presence of cats with type $\mathrm{AB}$ and the absence of type $\mathrm{B}$ indicate the peculiarity of the chosen population. These data may serve to alert the likelihood of adverse transfusion reactions and the occurrence of neonatal isoerythrolysis, and contribute to the selection of donors in an animal blood bank.
\end{abstract}

Keywords: erythrocyte antigen; immunohematology; system AB.

\section{Introdução}

Os grupos sanguíneos são definidos pela presença de antígenos espécie-específicos determinados pelas diferentes formas de ácido neuramínico presentes nos glicolipídeos e glicoproteínas da membrana dos eritrócitos, detectados por meio de testes sorológicos de aglutinação como a tipagem sanguínea (Andrews et al., 1992; Lanevschi e Wardrop, 2001; Giger et al., 2005).

A hemoterapia veterinária é considerada uma ferramenta diagnóstica imprescindível, pois a identificação da tipagem sanguínea em felinos pode evitar a ocorrência da isoeritrólise neonatal e auxiliar na prevenção de reações transfusionais, 
uma vez que a transfusão sanguínea é um procedimento recorrente na prática clínica veterinária de felinos domésticos (Kerl e Hohenhaus, 1993; Barfield e Adamantos, 2001; Hohenhaus, 2004).

O sistema sanguíneo $\mathrm{AB}$ dos felinos descrito Auer e Bell (1981), que os agrupou em três tipos: A, B e AB. Os tipos sanguíneos apresentam distribuição e frequência de acordo com a região geográfica e raça do animal. O tipo A é mais comum, com prevalência de até $100 \%$ em algumas populações (Hohenhaus, 2004), enquanto que a frequência do tipo $B$ diversifica-se entre as raças e o tipo AB é raramente descrito (Giger et al., 1991, Silva et al., 2016).

Os felinos apresentam anticorpos denominados aloanticorpos, que reagem contra o antígeno eritrocitário do tipo sanguíneo incompatível (Auer e Bell, 1981; Griot-Wenk e Giger, 1995). Os aloanticorpos são clinicamente importantes, pois condicionam a gravidade e a ocorrência da isoeritrólise neonatal e a severidade das reações transfusionais (Gurkan et al., 2005).

Os gatos do grupo sanguíneo B, geralmente, possuem elevadas titulações de aloanticorpos antiA, e manifestam reações transfusionais fatais ao receber o sangue de animais tipo A. Estes, por sua vez, frequentemente apresentam baixas concentrações de anticorpos anti-B e por isso desenvolvem sinais brandos de anemia hemolítica aguda ao receberem sangue do tipo B (Knottenbelt, 2002).

Felinos do grupo sanguíneo $A B$ não possuem aloanticorpos, mas devem preferencialmente receber sangue tipo $\mathrm{AB}$, contudo na indisponibilidade de doadores com o mesmo tipo sanguíneo, a transfusão pode ser realizada com sangue de animais tipo A, pois a maioria destes gatos apresentam níveis baixos de anticorpos antiB (Auer e Bell, 1983; Bucheler e Giger, 1993). Associado a tipagem sanguínea, é imprescindível realizar a prova de compatibilidade já que a presença de um resultado positivo na reação cruzada pode não ser tão específico quando o tipo sanguíneo $\mathrm{AB}$ está envolvido, uma vez que gatos $\mathrm{AB}$ não possuem aloanticorpos naturais, consequentemente pode ocorrer aglutinação na prova menor se testados com tipos A e B como doadores e na prova maior como receptores (Knottenbelt et al., 1999b).

Segundo Knottenbelt (2002), filhotes com tipos sanguíneos $\mathrm{A}$ e $\mathrm{AB}$ nascidos de gatas do tipo B apresentam considerável risco para o desenvolvimento de isoeritrólise neonatal na amamentação, em consequência da presença de anticorpos anti-A no colostro. A vida média dos eritrócitos após a transfusão dura aproximadamente de quatro a cinco semanas na corrente sanguínea do receptor, em contrapartida, quando incompatíveis, as hemácias podem durar apenas poucas horas a alguns dias ou até mesmo desencadear uma reação transfusional hemolítica imunomediada aguda severa.

Estudos divergem sobre a frequência fenotípica dos grupos sanguíneos em felinos domésticos e admitem que o conhecimento da distribuição dos tipos sanguíneos de uma população regional aumenta a segurança na aplicação clínica da hemoterapia, incrementando o sucesso terapêutico (Silva et al., 2016). Além disso, com o aumento do número de bancos de sangue animal esta informação pode contribuir para um melhor planejamento dos derivados sanguíneos que serão utilizados no atendimento de pacientes felinos (Knottenbelt, 2002)

Vale destacar também que o procedimento de tipificação sanguínea dos reprodutores é essencial, para prevenir a isoeritrólise neonatal, garantindo principalmente a deteç̧ão de fêmeas tipo B (Arikan et al., 2006). Diante do exposto, o objetivo com este trabalho foi determinar as frequências dos tipos sanguíneos, do sistema $A B$, de gatos domésticos sem raça definida na cidade de Campo Grande, Mato Grosso do Sul, Brasil.

\section{Material e métodos}

O estudo foi realizado com 202 gatos domésticos (Felis catus), sem raça definida, provenientes do Centro de Controle de Zoonoses (CCZ) do município de Campo Grande, Mato Grosso do Sul (MS), Brasil.

Foram selecionados animais encaminhados para procedimento cirúrgico eletivo de ovariosalpingohisterectomia e orquiectomia, com peso corpóreo superior a 1,5 quilos, independente de sexo e idade. Os gatos que apresentavam histórico recente de doença grave, uso de medicamentos, submetidos à hemoterapia prévia ou fêmeas gestantes foram excluídos do experimento. Dados epidemiológicos e clínicos do animal, e bairro de residência do proprietário foram obtidos a partir da ficha de anamnese disponibilizada pelo CCZ.

De cada animal foram coletados $4 \mathrm{~mL}$ de sangue por punção da veia jugular e distribuídos em tubo com ácido etilenodiaminotetracético 
dipotássico, (EDTA K 2) para a tipagem sanguínea e tubo sem aditivos para obtenção do soro para a tipagem reversa. Os procedimentos laboratoriais foram conduzidos no Laboratório de Patologia Clínica do Hospital Veterinário da Universidade Federal de Mato Grosso do Sul (UFMS).

A tipagem sanguínea foi realizada imediatamente após a coleta, por meio do teste de hemaglutinação em tubo de ensaio. As amostras de sangue com EDTA foram centrifugadas a $400 \mathrm{~g}$ de força durante 5 minutos para separação do plasma. O plasma e a capa leucocitária foram retirados com pipeta. Após o fracionamento celular, o concentrado de eritrócitos foi transferido com o auxílio de uma pipeta para um tubo de ensaio e submetido a três lavagens consecutivas com PBS (tampão fosfato $\mathrm{pH} 7,2$ ) e centrifugação a $1972 \mathrm{~g}$ de força durante três minutos. Logo após a última lavagem, uma suspensão de hemácias a $5 \%$ foi preparada $(50 \mu \mathrm{L}$ do concentrado de eritrócitos diluídos em $1 \mathrm{~mL}$ de PBS).

Posteriormente, foram dispostos em três tubos de ensaio devidamente identificados: $50 \mu \mathrm{L}$ de PBS (tubo C - controle), $50 \mu \mathrm{L}$ do soro anti-A (tubo A) e $50 \mu \mathrm{L}$ da solução anti-B (tubo B) preparada com Triticum vulgaris $(8 \mu \mathrm{g} / \mathrm{mL})$ e PBS. Triticum vulgaris corresponde a uma lectina de germe de trigo, que induz aglutinação de células tipo B.

Em seguida foram adicionados, a cada tubo, $25 \mu \mathrm{L}$ da suspensão de eritrócitos e homogeneizados suavemente. Após 15 minutos de incubação à temperatura ambiente, os tubos foram centrifugados por 15 segundos a $2086 \mathrm{~g}$ de força. Por fim, a leitura do resultado foi realizada ressuspendendo as células com leves movimentos no tubo e observando-se a presença de aglutinação. Para a confirmação dos tipos sanguíneos $\mathrm{B}$ e $\mathrm{AB}$ a tipagem reversa foi realizada com o método similar descrito para a tipagem sanguínea, por meio da incubação do soro de cada animal com suspensão de células de gatos do tipo A e B conhecidos.

Os dados obtidos foram submetidos à análise de frequência absoluta e relativa dos diferentes antígenos eritrocitários sobre a população estudada. A frequência dos tipos sanguíneos foi distribuída de acordo com a região de moradia do proprietário. A cidade de Campo Grande é dividida em sete regiões de acordo com o Instituto Municipal de Planejamento Urbano: Anhanduizinho, Bandeira, Centro, Imbirussu, Lagoa, Prosa e Segredo (PMCG, 2008).

\section{Resultados}

Dos felinos domésticos mestiços avaliados $61 \%$ (124/202) eram fêmeas e 39\% (78/202) machos com idade variando entre seis meses e sete anos. O tipo sanguíneo A foi predominante, com frequência de 98,5\% (199/202), três animais $(1,5 \%)$ apresentaram o tipo $\mathrm{AB}$ e o tipo $\mathrm{B}$ não foi encontrado.

A Tabela 1 exibe os dados da hemaglutinação e frequência dos tipos sanguíneos do sistema $\mathrm{AB}$ em gatos mestiços observados no estudo.

A distribuição da origem dos animais estudados, de acordo com a região administrativa do município de Campo Grande pode ser observada no Tabela 2.

Tabela 1. Resultados da hemaglutinação e frequência dos tipos sanguíneos do Sistema AB de gatos domésticos mestiços em Campo Grande, Brasil.

\begin{tabular}{cccc}
\hline Tipo sanguíneo* & $\%(\mathrm{n})$ & $\begin{array}{c}\text { Aglutinação com lectina Triticum } \\
\text { vulgaris }\end{array}$ & $\begin{array}{c}\text { Aglutinação com Soro Anti- } \\
\text { A }\end{array}$ \\
\hline A & $98,5(199)$ & Negativo & Positivo \\
B & $0,0(0)$ & Positivo & Negativo \\
AB & $1,5(3)$ & Positivo & Positivo \\
\hline
\end{tabular}

\footnotetext{
*Tipos $\mathrm{B}$ e $\mathrm{AB}$ foram confirmados com a tipagem reversa.
} 
Tabela 2. Distribuição das frequências dos tipos sanguíneos de gatos domésticos mestiços de acordo com as regiões administrativas de Campo Grande, MS.

\begin{tabular}{cccc}
\hline Região & \multicolumn{3}{c}{ Tipos Sanguíneos } \\
\cline { 2 - 4 } & Tipo A & Tipo B & Tipo AB \\
& $\mathrm{N}=199(\%)$ & $\mathrm{N}=0(\%)$ & $\mathrm{N}=3(\%)$ \\
\hline Anhanduizinho & $45(22,3)$ & - & $01(0,5)$ \\
Bandeira & $40(19,8)$ & - & $01(0,5)$ \\
Centro & $13(06,4)$ & - & - \\
Imbirussu & $24(11,9)$ & - & - \\
Lagoa & $37(18,3)$ & - & $01(0,5)$ \\
Mata do Segredo & $28(13,9)$ & - & - \\
Prosa & $12(05,9)$ & - & \\
\hline
\end{tabular}

Tabela 3. Frequência dos tipos sanguíneos em gatos domésticos sem definição racial.

\begin{tabular}{|c|c|c|c|c|c|}
\hline \multicolumn{6}{|c|}{ Frequência dos tipos sanguíneos } \\
\hline Localidade & $\mathrm{N}$ & $\begin{array}{c}\text { Tipo A } \\
(\%)\end{array}$ & $\begin{array}{c}\text { Tipo B } \\
(\%)\end{array}$ & $\begin{array}{c}\text { Tipo AB } \\
(\%)\end{array}$ & Referências \\
\hline \multicolumn{6}{|l|}{ Brasil } \\
\hline Porto Alegre & 105 & 97,1 & 1,9 & 1,0 & Guerra et al. (2007) \\
\hline Porto Alegre & 100 & 97,0 & 3,0 & 0,0 & Lacerda et al. (2008) \\
\hline Rio de Janeiro & 48 & 93,75 & 0,0 & 6,25 & Vilar (2006) \\
\hline Rio de Janeiro & 172 & 94,8 & 2,9 & 2,3 & Medeiros et al. (2008) \\
\hline Rio de Janeiro & 86 & 98,8 & 1,2 & 0,0 & Pinto et al. (2016) \\
\hline Paraíba & 164 & 98,1 & 1,21 & 0,69 & Mendes et al. (2013) \\
\hline Campo Grande & 202 & 98,5 & 0,0 & 1,5 & Presente estudo \\
\hline Estados Unidos & 1072 & 99,7 & 0,3 & 0,0 & Giger et al. (1991) \\
\hline Canadá & 178 & 94,4 & 5,0 & 0,6 & Fosset e Blais (2014) \\
\hline Austrália & 187 & 62,0 & 36,0 & 1,6 & Malik et al. (2005) \\
\hline Japão & 261 & 90,03 & 0,77 & 9,2 & Ejima et al. (1986) \\
\hline China & 262 & 88,2 & 11,4 & 0,4 & Zheng et al. (2011) \\
\hline \multicolumn{6}{|l|}{ Portugal } \\
\hline Norte & 159 & 89,3 & 4,4 & 6,3 & Silvestre-Ferreira et al. (2001) \\
\hline Portugal & 515 & 97,5 & 2,1 & 0,4 & Marques et al. (2011) \\
\hline Grécia & 207 & 78,3 & 20,3 & 1,4 & Mylonakis et al. (2001) \\
\hline Escócia & 139 & 87,1 & 7,9 & 5,0 & Knottenbelt et al. (1999a) \\
\hline Finlândia & 61 & 100 & 0 & 0 & Giger et al. (1992) \\
\hline Irlanda & 137 & 84,7 & 14,6 & 0,7 & Juvet et al. (2011) \\
\hline Inglaterra & 105 & 67,6 & 30,5 & 1,9 & Forcada et al. (2007) \\
\hline Espanha & 100 & 94,0 & 5,0 & 1,0 & Gopegui et al. (2004) \\
\hline Turquia & 301 & 73,1 & 24,6 & 2,3 & Arikan et al. (2006) \\
\hline
\end{tabular}

\section{Discussão}

A avaliação dos resultados dos 202 felinos domésticos, do município de Campo Grande - MS, para detectar a distribuição da frequência dos diversos grupos sanguíneos, mostrou uma diferença entre os fenótipos A, B e AB. Ao comparar a distribuição do sistema sanguíneo $\mathrm{AB}$ foi verificado que $199(98,5 \%)$ indivíduos eram do grupo sanguíneo $\mathrm{A}$, três $(1,5 \%)$ do grupo $\mathrm{AB}$ e nenhum animal do grupo $\mathrm{B}$ foi identificado, esses dados assemelham-se aos encontrados por Silva et al. (2016).

Embora, em diversos países, a frequência dos tipos sanguíneos em felinos domésticos sem raça definida tenha sido tipificada, no Brasil (Tabela 3) estes dados ainda são restritos as regiões Sul, Sudeste e Nordeste (Vilar, 2006; Guerra et al., 2007; Lacerda et al., 2008; Medeiros et al., 2008; Mendes et al., 2013; Pinto et al., 2016). Marques et al. (2011) e Silva et al. (2016) afirmaram que a 
caracterização dos tipos sanguíneos, de acordo com a área geográfica, em uma população, pode auxiliar na predição da probabilidade de reações adversas em terapias transfusionais. Além disso, possibilita o direcionamento da demanda dos produtos sanguíneos oferecidos e a seleção de doadores em bancos de sangue animal.

Neste estudo observou-se um predomínio do tipo sanguíneo A corroborando os estudos conduzidos com gatos domésticos no Brasil e na Europa (Giger et al., 1992; Ruiz de Gopegui et al., 2004; Vilar, 2006; Lacerda et al., 2008; Medeiros et al., 2008; Marques et al., 2011; Juvet et al., 2011; Mendes et al., 2013; Pinto et al., 2016) como pode ser constatado na Tabela 3. Apesar do predomínio mundial do antígeno eritrocitário $\mathrm{A}$, a frequência varia geograficamente entre $60,9 \%$ a $100 \%$ (Giger et al., 1992; Mylonakis et al., 2001; Malik et al., 2005; Arikan et al., 2006; Forcada et al., 2007; Zheng et al., 2011; Fosset e Blais, 2014). A maior prevalência deste tipo sanguíneo, provavelmente, resulta do padrão da hereditariedade do sistema $\mathrm{AB}$ em felinos, que é regulado por um conjunto de três genes alélicos $(\mathrm{A}, \mathrm{B}, \mathrm{AB})$, dos quais $\mathrm{A}$ e $\mathrm{AB}$ são dominantes sobre $\mathrm{B}$, enquanto $\mathrm{AB}$ parece ser recessivo para $\mathrm{A}$, além da predominância de gatos homozigotos para o alelo A (Giger et al., 1991; Griot-Wenk et al., 1996).

A ausência do tipo sanguíneo B nos animais avaliados no presente estudo pode ser considerada incomum ao comparar dados de outras investigações, com o mesmo tipo de população (Guerra et al. 2007; Lacerda et al. 2008; Medeiros et al. 2008; Mendes et al. 2013). Em países como Austrália, China, Grécia, Inglaterra e Turquia a frequência do tipo B variou de 11,4 a $36 \%$ (Tabela 3) (Mylonakis et al., 2001; Malik et al., 2005; Arikan et al., 2006; Forcada et al., 2007; Zheng et al., 2011). No Brasil esses índices variaram entre 0 $\%$ a 3,0 \% (Tabela 3) (Vilar, 2006; Guerra et al., 2007; Lacerda et al., 2008; Medeiros et al., 2008; Mendes et al., 2013; Pinto et al., 2016). Esta diversidade provavelmente está associada ao efeito da seleção de progenitores em cada região o que contribui para maior ou menor incidência de fenótipos homozigotos recessivos nas populações (Auer e Bell, 1981; Gurkan et al., 2005; Forcada et al., 2007) reforçando a necessidade de conhecimentos regionalizados dos dados.

$\mathrm{O}$ tipo sanguíneo $\mathrm{AB}$ foi identificado em três animais, o que equivaleu a $1,5 \%$ da população estudada. Este índice embora inferior aos observados em outros países (Ejima et al., 1986;
Knottenbelt et al., 1999a; Silvestre-Ferreira et al., 2004) aproxima-se dos valores obtidos em algumas regiões do Brasil (Vilar, 2006). A presença de gatos do tipo sanguíneo $A B$ e a ausência do tipo $B$ observados nos animais do estudo podem refletir um processo de deriva genética aleatória, resultante do aumento na frequência do alelo $\mathrm{AB}$ ou do cruzamento com raças que apresentam elevada prevalência deste tipo sanguíneo (Knottenbelt et al., 1999a).

Segundo Giger et al. (1991) felinos domésticos com o tipo sanguíneo $\mathrm{AB}$ somente são detectados quando existem animais tipo $\mathrm{B}$ na população. Porém, estudo em população de gatos de raça, com frequência elevada de sangue do tipo $\mathrm{B}$, não comprovou a presença de animais com tipo sanguíneo AB (Arikan et al., 2003) e levantou a especulação de que o sangue $A B$ deriva de um terceiro alelo que permite a expressão codominante de antígenos A e B (Griot-Wenk et al., 1996).

\section{Conclusão}

O Grupo sanguíneo A foi o fenótipo mais frequente em Campo Grande, MS, contudo destacam-se a presença de gatos com tipo sanguíneo $\mathrm{AB}$ e ausência do tipo sanguíneo $\mathrm{B}$ uma peculiaridade regional dessa população. Cabe ressaltar a aplicabilidade dos dados obtidos na conduta médico-veterinária, pois, alerta sobre a probabilidade de reações transfusionais adversas e ocorrência de isoeritrólise neonatal, além de contribuir para a seleção de doadores e demanda de reserva em banco de sangue animal.

\section{Conflito de Interesse}

Os autores declaram não existir conflito de interesse.

\section{Comitê de Ética}

O experimento foi aprovado pela Comissão de Ética no Uso de Animais da Universidade Federal de Mato Grosso do Sul (UFMS), sob protocolo número 688/2015.

\section{Agradecimentos}

A Fundação de Apoio ao Desenvolvimento do Ensino, Ciência e Tecnologia do Estado de Mato Grosso do Sul (FUNDECT) pelo auxílio financeiro.

\section{Referências}

Andrews, G.A.; Chavey, P.S.; Smith, J.E.; Rich, L. $\mathrm{N}$-glycolylneuraminicacid and $\mathrm{N}$ - 
acetylneuraminic acid define feline blood group A and B antigens. Blood, 79(9): 24852491, 1992.

Arikan, S.; Gurkan, M.; Ozaytekin, E.; Dodurka, T.; Giger, U. Frequencies of blood type A, B and $\mathrm{AB}$ in non-pedigree domestic cats in Turkey. Journal of Small Animal Practice, 47(1):10-13, 2006.

Arikan, S.; Duru, S. Y.; Gurkan, M.; Agaoglu, Z. T.; Giger, U. Blood type A and B frequencies in Turkish Van and Angora cats in Turkey. Transboundary and Emerging Diseases, 50(6):303-306, 2003.

Auer, L.; Bell, K. Transfusion reactions in cats due to $\mathrm{AB}$ blood group incompatibility. Research in Veterinary Science, 35(2):145-152, 1983.

Auer, L.; Bell, K. The AB blood group system of cats. Animal Blood Groups and Biochemical Genetics, 12(3):287-297, 1981.

Barfield, D.; Adamantos, S. Feline blood transfusions: A pinker shade of pale. Journal of Feline Medicine and Surgery, 13(1):1123, 2011.

Bücheler, J.; Giger, U.; Alloantibodies against A and B blood types in cats. Veterinary Immunology and Immunopathology, 38(34):283-295, 1993.

Ejima, H.; Kurokawa, K.; Ikemoto, S. Feline red blood cell groups detected by naturally occurring isoantibody. The Japanese Journal of Veterinary Science, 48(5): 971976, 1986.

Forcada, Y.; Guitian, J.; Gibson, G. Frequencies of feline blood types at a referral hospital in the south east of England. Journal of Small Animal Practice, 48(10): 570-573, 2007.

Fosset, F.T.J.; Blais, M.C. Prevalence of feline blood groups in the Montreal area of Quebec, Canada. The Canadian Veterinary Journal, 55(1):1225, 2014.

Giger, U.; Bucheler, J.; Patterson, D.F. Frequency and inheritance of $\mathrm{A}$ and $\mathrm{B}$ blood types in feline breeds of the United States. Journal of Heredity, 82(1):15-20, 1991.

Giger, U.; Gorman, N.T.; Hubler, M.; Leidinger, J.I.; Leidinger, E.F.; Lubas, G.; Niini, T.; Slappendel, R.J. Frequencies of feline A and B blood types in Europe. Animal Genetics, 23(1): 17-18, 1992.

Giger, U.; Stieger, K.; Palos, H. Comparison of various canine blood-typing methods. American Journal of Veterinary Research, 66 (8): 1386-1392, 2005.
Griot-Wenk, M.E.; Giger, U. Feline transfusion medicine: blood types and their clinical importance. Veterinary Clinics of North America: Small Animal Practice, 25(6): 1305-1322, 1995.

Griot-Wenk, M.E.; Callan, M.B.; Casal, M.L.; Chisholm-Chait, A.; Spitalnik, S.L.; Patterson, D.F.; Giger, U. Blood type AB in the feline $\mathrm{AB}$ blood group system. American Journal of Veterinary Research, 57(10):1438-1442, 1996.

Guerra, T.A.; Lacerda, L.A.; Oliveira, S.T.; Esteves, V.S.; González, F.H.D. Tipagem sanguínea em felinos: 148 gatos domésticos na rotina laboratorial do Lacvet -UFGRGS. Acta Scientiae Veterinariae, 35(2):573-574, 2007.

Gurkan, M.; Arikan, Ş.; Ozaytekin, E.; Dodurka, T. Titres of alloantibodies against A and B blood types in non-pedigree domestic cats in Turkey: assessing the transfusion reaction risk. Journal of Feline Medicine \& Surgery, 7(5):301-305, 2005.

Hohenhaus, A.E. Importance of blood groups and blood group antibodies in companion animals. Transfusion Medicine Reviews, 18(2):117126, 2004.

Juvet, F.; Brennan, S.; Mooney, C.T. Assessment of feline blood for transfusion purposes in the Dublin area of Ireland. The Veterinary Record, 168(13):352-352, 2011.

Kerl, M.E.; Hohenhaus, A.E. Packed red blood cell transfusions in dogs: 131 cases (1989). Journal of the American Veterinary Medical Association, 202(9):1495-1499, 1993.

Knottenbelt, C.M.; Addie, D.D.; Day, M.J., Mackin, A.J. Determination of the prevalence of feline blood types in the UK. Journal of Small Animal Practice, 40(3): 115-118, 1999a.

Knottenbelt, C.M.; Day, M.J.; Cripps, P.J.; Mackin, A.J. Measurement of titres of naturally occurring alloantibodies against feline blood group antigens in the UK. Journal of Small Animal Practice, 40(8): 365-370, 1999b.

Knottenbelt, C.M. The feline AB blood group system and its importance in transfusion medicine. Journal of Feline Medicine and Surgery, 4(2): 69-76, 2002.

Lacerda, L.A.; Oliveira, S.T.; Guerra, T.A.; Stein, G. G., González, F. H. D. Prevalência dos 
tipos sanguíneos A, B e AB em gatos domésticos mestiços da cidade de Porto Alegre, Rio Grande do Sul, Brasil. Brazilian Journal of Veterinary Research and Animal Science, 45:46-53, 2008.

Lanevschi, A.; Wardrop, K.J. Principles of transfusion medicine in small animals. The Canadian Veterinary Journal, 42(6): 447, 2001.

Malik, R.; Griffin, D.L.; White, J.D.; Rozmanec, M.; Tisdall, P.L.C., Foster, S.F., Nicholas, F.W. The prevalence of feline A/B blood types in the Sydney region. Australian Veterinary Journal, 83(1-2):38-44, 2005.

Marques, C.; Ferreira, M.; Gomes, J. F.; Leitão, N.; Costa, M.; Serra, P.; Pomba, C.F. Frequency of blood type $\mathrm{A}, \mathrm{B}$, and $\mathrm{AB}$ in 515 domestic shorthair cats from the Lisbon area. Veterinary Clinical Pathology, 40(2):185187, 2011.

Medeiros, M.A.; Soares, A.M.; Alviano, D.S.; Ejzemberg, R.; Da Silva, M.H., Almosny, N.R. Frequencies of feline blood types in the Rio de Janeiro area of Brazil. Veterinary Clinical Pathology, 37(3): 272-276, 2008.

Mendes, R.D.S.; Gurjão, T.A.; Souza, A.P.; Lacerda, L.D.A.; Silva, R.M. Frequência dos antígenos eritrocitários do sistema $\mathrm{AB}$ em felinos domésticos no estado da Paraíba. Pesquisa Veterinária Brasileira, 33(6):780784, 2013.

Mylonakis, M.E.; Koutinas, A.F.; Saridomichelakis, M.; Leontidis, L.; Papadogiannakis, M.; Plevraki, K.
Determination of the prevalence of blood types in the non-pedigree feline population in Greece. Veterinary Record, 149(7): 213214, 2001.

Pinto, A.B.T.; Medeiros, M.A.S.; Jardim, M.P.B.; Albernaz, A.P. Frequências dos tipos sanguíneos e títulos dos aloanticorpos em felinos domésticos. Ciência Animal Brasileira, 17(2):225-235, 2016.

PMCG. Prefeitura Municipal de Campo Grande. Instituto Municipal de Planejamento Urbano PLANURB - Perfil Socioeconômico de Campo Grande. 22. ed. 2015. 382p.

Silva, P.B.; Monteiro, M.V.B.; Silva, R.R.; Albuquerque, M.R.; Pereira, A.C.A.; Carreira, A. S.; Monteiro, F.O.B. Frequência dos tipos sanguíneos de gatos domésticos oriundos do estado do Pará, Brasil. Acta Veterinaria Brasilica, 10 (4): 378-382, 2016.

Silvestre-Ferreira, A.C.; Pastor, J.; Almeida, O.; Montoya A. Frequencies of feline blood types in northern Portugal. Veterinary Clinical Pathology, 33(4): 240-243, 2004.

Vilar, T.D. Tipagem sanguínea em gatos - Estudo da prevalência dos antígenos eritrocitários do sistema $\mathrm{AB}$ felino utilizando metodologia gelteste no estado do Rio de Janeiro, Brasil. Nosso Clínico, 54(28):31, 2006.

Zheng, L.; Zhong, Y.; Shi Z.; Giger, U. Frequencies of blood types $\mathrm{A}, \mathrm{B}$, and $\mathrm{AB}$ in non-pedigree domestic cats in Beijing. Veterinary Clinical Pathology, 40(4):513$517,2011$. 